\title{
Lesson of the month 1: A rash decision
}

\author{
Authors: Catherine L Sedgwick, ${ }^{A}$ Peter E Hall, ${ }^{\mathrm{B}}$ Abarna Ratnarajah, ${ }^{\mathrm{C}}$ Janakan Natkunarajah ${ }^{\mathrm{D}}$ and Louise Hogh ${ }^{\mathrm{E}}$
}

Cellulitis is a common condition and several mimics exist which should be considered in patients who fail to respond to antibiotics. We describe the case of a patient with anaplastic large cell lymphoma masquerading as a lower leg cellulitis. The patient had failed to respond to intravenous antibiotics and a skin biopsy confirmed her diagnosis. She received radical radiotherapy to the lower leg but later developed shortness of breath and was identified to have pulmonary infiltration of the lymphoma. She died shortly afterwards from lobar pneumonia. This case highlights the importance of regularly reassessing patients with suspected cellulitis and considering alternative diagnoses in cases that fail to respond to treatment.

KEYWORDS: Cellulitis, lymphoma, skin biopsy

\section{Case history}

An 81-year-old Caucasian female was referred to A\&E by her GP with a seven-day history of a tender left lower leg with associated swelling, which had failed to respond to oral or IV antibiotics. The patient was otherwise well, but did recall sustaining a small laceration to her left lower leg on a car door one month earlier. She had no history of travel.

Significant past medical history included a left total knee replacement for primary osteoarthritis seven months earlier. She also suffered from hypertension, atrial fibrillation, diverticulosis and transitional cell carcinoma of the bladder (treated with cystodiathermy). Her medications included aspirin, ramipril, bisoprolol, bendroflumethiazide and cocodamol. She was a retired teacher who lived alone, walked with a stick and was otherwise independent. She had never smoked and drank two units of alcohol per day.

On admission she was haemodynamically stable. Examination of her left lower leg revealed well-demarcated, tender erythema

Authors: Afoundation year 1 doctor, Kingston Hospital NHS Foundation Trust, Kingston upon Thames, UK; ${ }^{B}$ core medical trainee year 1, Kingston Hospital NHS Foundation Trust, Kingston upon Thames, UK; ' CST5 (acute medicine), Kingston Hospital NHS Foundation Trust, Kingston upon Thames, UK; ${ }^{D}$ Consultant dermatologist, Kingston Hospital NHS Foundation Trust, Kingston upon Thames, UK; Econsultant geriatrician, Kingston Hospital NHS Foundation Trust, Kingston upon Thames, UK on the anterior shin and the presence of a small skin-coloured nodule. There was significant pitting oedema and superficial skin erosions were present. Peripheral pulses were palpable and a Doppler ultrasound scan excluded a deep vein thrombosis.

The patient's initial blood tests revealed a normocytic anaemia with an elevated C-reactive protein. Blood cultures and skin swabs were normal, as were a fasting blood glucose and HbAlc. The patient was found to be HIV negative.

In view of the clinical signs, the patient was treated for left leg cellulitis with IV teicoplanin. Her inflammatory markers continued to deteriorate and she complained of increasing pain and swelling in her left leg. Her antibiotic dose was increased and fusidic acid was added. By day 15 , the patient's antibiotics were switched to tazosin.

A dermatology opinion was sought, given the patient's failure to respond to intravenous therapy and her increasing leg oedema. Examination revealed multiple erythematous dermal nodules over the left knee scar site and around the left shin, with associated leg oedema (Fig 1). A cutaneous lymphoma of the leg was suspected and an urgent skin biopsy was arranged. The biopsy revealed a dense infiltrate of large malignant cells with apoptosis and excess mitotic activity in the mid and deeper layers of the dermis, consistent with a high-grade large cell lymphoma. A CT scan of the neck, chest, abdomen and pelvis showed no evidence of systemic involvement of lymphoma. A diagnosis of $\mathrm{CD}^{2} 0^{+}$non-Hodgkin's anaplastic large cell lymphoma was made and the patient was referred to a tertiary centre, where she received radical radiotherapy to the left lower leg (30 Gy).

Unfortunately the patient re-presented two months later with shortness of breath and lethargy. A chest X-ray showed a moderately sized, right-sided pleural effusion with right upper and middle lobe collapse. A CT pulmonary angiogram excluded a pulmonary embolism but identified large volume mediastinal lymphadenopathy, pleural effusion, multiple nodular changes and coarse interlobular thickening, consistent with pulmonary involvement of the lymphoma. Sadly the patient died a week later of lobar pneumonia.

\section{Discussion}

\section{Cellulitis}

Cellulitis is misdiagnosed in $13-28 \%$ of patients labelled as having the condition. ${ }^{1,2}$ In patients who do not respond to antimicrobial therapy, alternative diagnoses should be considered. There is a wide differential of non-infectious mimics, ranging from common conditions, such as stasis 


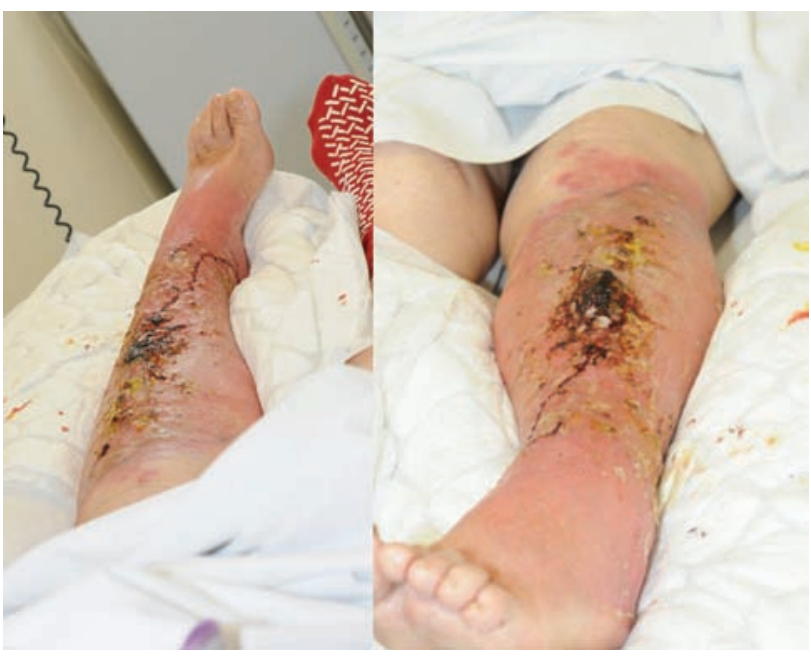

Fig 1. Multiple erythematous dermal nodules and oedema of the left lower leg, after two weeks of antibiotic therapy.

dermatitis and deep vein thrombosis, to rarer conditions including immunological disorders and malignancy. ${ }^{3,4}$ Signs and symptoms of a non-infectious cause can include a bilateral distribution, a lack of systemic disturbance, such as fever, and a chronic time course.

\section{Primary cutaneous lymphomas}

Primary cutaneous lymphomas are non-Hodgkin's lymphomas (NHLs), for which there is no evidence of extracutaneous disease at the time of diagnosis. They account for $5 \%$ of all NHLs with an annual incidence of approximately 1 per 100,000 person-years. ${ }^{5}$ T-cell subtypes make up $70 \%$ of cutaneous lymphomas with B-cell subtypes representing $30 \%$. Both can be further subdivided on the basis of histological, immunophenotypic and clinical findings according to the WHO-European Organisation for Research and Treatment of Cancer (EORTC) classification. ${ }^{6}$ Primary cutaneous lymphomas behave in a different manner to systemic lymphomas which secondarily affect the skin and therefore require alternative treatments.

\section{CD30+ lymphoproliferative disorders}

$\mathrm{CD} 30^{+}$lymphoproliferative disorders are T-cell lymphomas that can be sub-divided into primary cutaneous anaplastic large cell lymphoma and lymphomatoid papulosis according to the WHO-EORTC classification. ${ }^{6}$ Based on overlapping characteristics, these two conditions are considered to be a spectrum of $\mathrm{CD} 0^{+}$disease. ${ }^{7}$ Anaplastic large cell lymphoma accounts for $8 \%$ of cutaneous lymphomas. ${ }^{6,7}$

Data from cutaneous lymphoma registries have shown that cutaneous anaplastic large cell lymphoma affects males:females in a 2-3:1 ratio with a mean age at diagnosis of 60 years old. 6,7 In approximately $80 \%$ of cases, the lymphoma is either solitary or localised to one skin area. Multifocal lesions are seen in 20\% of cases. Ulceration and crusting is common. Extracutaneous disease occurs in approximately $10 \%$ of cases.

The treatment modality for solitary or localised disease is either excision or radiotherapy. ${ }^{6,7}$ Low-dose methotrexate can be considered for multifocal lesions. For disseminated extracutaneous disease, treatment with doxorubicin-based multiagent chemotherapy is recommended. Spontaneous partial or complete regression can occur in up to $40 \%$ of cases (at diagnosis or relapse). If complete regression occurs, then no further treatment is required. Skin relapses after treatment are common, but may initially be observed for spontaneous regression and do not represent aggressive disease.

Five-year survival rates are greater than 95\%. Interestingly, no risk factors for tumour progression or dissemination have been identified, including age of onset, multifocal skin lesions and one draining lymph node station involvement. ${ }^{7}$

\section{References}

1 David CV, Chira S, Eells SJ et al. Diagnostic accuracy in patients admitted to hospitals with cellulitis. Dermatol Online J 2011;17:1.

2 Hepburn MJ, Dooley DP, Ellis MW. Alternative diagnoses that often mimic cellulitis. Am Fam Physician 2003;67:2471.

3 Keller EC, Tomecki KJ, Alraies MC. Distinguishing cellulitis from its mimics. Cleve Clin J Med 2012;79:547-52.

4 Falagas ME, Vergidis PI. Narrative review: diseases that masquerade as infectious cellulitis. Ann Intern Med 2005;142:47-55.

5 Groves FD, Linet MS, Travis LB, Devesa SS. Cancer surveillance series: non-Hodgkin's lymphoma incidence by histologic subtype in the United States from 1978 through 1995. J Natl Cancer Inst 2000;92:1240-51.

6 Willemze R, Jaffe ES, Burg G et al. WHO-EORTC classification for cutaneous lymphomas. Blood 2005;105:3768-85.

7 Bekkenk MW, Geelen FA, van Voorst Vader PC et al. Primary and secondary cutaneous $\mathrm{CD} 30(+)$ lymphoproliferative disorders: a report from the Dutch Cutaneous Lymphoma Group on the longterm follow-up data of 219 patients and guidelines for diagnosis and treatment. Blood 2000;95:3653-61.

Address for correspondence: Dr C Sedgwick, Croydon University Hospital, 530 London Road, Croydon CR7 7YE, UK. Email: catherine.sedgwick@ymail.com 